\title{
Role of electron-electron interference in ultrafast time-resolved imaging of electronic wavepackets
}

\author{
Gopal Dixit ${ }^{1, *}$ and Robin Santra ${ }^{1,2,+\dagger}$ \\ ${ }^{1}$ Center for Free-Electron Laser Science, DESY, \\ Notkestrasse 85, D-22607 Hamburg, Germany \\ ${ }^{2}$ Department of Physics, University of Hamburg, D-20355 Hamburg, Germany
}

(Dated: March 13, 2013)

\begin{abstract}
Ultrafast time-resolved x-ray scattering is an emerging approach to image the dynamical evolution of the electronic charge distribution during complex chemical and biological processes in realspace and real-time. Recently, the differences between semiclassical and quantum-electrodynamical (QED) theory of light-matter interaction for scattering of ultrashort x-ray pulses from the electronic wavepacket were formally demonstrated and visually illustrated by scattering patterns calculated for an electronic wavepacket in atomic hydrogen [Proc. Natl. Acad. Sci. U.S.A., 109, 11636 (2012)]. In this work, we present a detailed analysis of time-resolved x-ray scattering from a sample containing a mixture of non-stationary and stationary electrons within both the theories. In a many-electron system, the role of scattering interference between a non-stationary and several stationary electrons to the total scattering signal is investigated. In general, QED and semiclassical theory provide different results for the contribution from the scattering interference, which depends on the energy resolution of the detector and the x-ray pulse duration. The present findings are demonstrated by means of a numerical example of x-ray TRI for an electronic wavepacket in helium. It is shown that the time-dependent scattering interference vanishes within semiclassical theory and the corresponding patterns are dominated by the scattering contribution from the timeindependent interference, whereas the time-dependent scattering interference contribution does not vanish in the QED theory and the patterns are dominated by the scattering contribution from the non-stationary electron scattering.
\end{abstract}

PACS numbers:

*gopal.dixit@cfel.de 
robin.santra@cfel.de 


\section{INTRODUCTION}

To fully understand the functionality and dynamic behavior of molecules, solids and complex biological systems, it is important to image the motion of electrons in real-time and in real-space. The motion of atoms within molecules and solids that is associated with chemical transformations occurs on the femtosecond ( $\left.1 \mathrm{fs}=10^{-15} \mathrm{~s}\right)$ timescale. The timescale of electronic motion, responsible for electron-hole dynamics and electron transfer processes in molecules can be even faster, on the order of attoseconds $\left(1\right.$ as $\left.=10^{-18} \mathrm{~s}\right)\lfloor 1$ 5]. The ultimate goal of the emerging field of time-resolved imaging (TRI) is to visualize electronic motion on an ultrafast timescale as electrons move in atoms, complex molecules or solids, as occurring for instance in photoinduced exciton dynamics, during bond formation and breakage, conformational changes and charge migration [ $6-9]$. With the tremendous advancement in technology for producing ultraintense and ultrashort x-ray pulses from novel light sources, it seems possible to obtain information about ultrafast dynamics of electrons. In extension of the concept of "molecular movies", which track the motion of atoms on fs timescale [10-13], ultrashort, tunable, and high-energy x-ray pulses from free-electron lasers (FEL) [14, 15], laser plasmas [16] and high-harmonic generation [17, 18] promise to provide "electronic movies" that take place on few fs to as timescale [19]. Recent breakthroughs make it possible to generate hard x-ray pulses of a few fs [14, 20], and pulse duration of 100 as can in principle be realized [21, 22]. Utilizing the remarkable properties of x-rays from FEL, several new insights have been gained about systems ranging from atoms [23, 24], molecules [25, 26], clusters [27], complex biomolecules [28, 29], to matter in extreme conditions [30, 31].

Since the discovery of x-rays [32], scattering of x-rays from matter has been used to unveil the structure of molecules, solids and biomolecules with atomic-scale spatial resolution [3337]. Also, scattering of x-rays from atoms and molecules has been proposed to gain insight about the excited electronic states of atomic and molecular systems [38, 39]. In order to image electronic motion in real-time and real-space with spatial and temporal resolutions of order $1 \AA$ and 1 fs, respectively, one can perform scattering of ultrashort x-ray pulses from the dynamically evolving electronic charge distribution. Pump-probe experiments are the most direct approach, where first a pump pulse induces the dynamics and then subsequently a probe pulse interrogates such induced dynamics. By varying the pump-probe time delay, one obtains a series of scattering patterns that serve to image the electronic motion with 
atomic-scale spatio-temporal resolution.

Recently, the theory of TRI from a non-stationary electronic system, using both the semiclassical and the quantum electrodynamical (QED) treatment of light-matter interaction, has been developed [40]. In a semiclassical theory of light-matter interaction, matter is treated quantum mechanically and light is treated classically. In such a situation, the timedependent Schrödinger equation is solved for the electrons together with Maxwell's equations for the light. By solving Maxwell's equations for given charge and current densities, the expression for the differential scattering probability (DSP) is obtained, which is a key quantity in x-ray scattering. According to the semiclassical theory, x-ray TRI would be expected to provide access to the instantaneous electron density of the non-stationary electronic system. On the other hand, in a consistent quantum theory of light-matter interaction, where both matter and light are treated quantum mechanically, x-ray TRI encodes the information about spatio-temporal density-density correlation. Both the theories have been applied to an electronic wavepacket prepared as a coherent superposition of eigenstates of atomic hydrogen and it has been shown that the scattering patterns obtained using both the theories differ drastically from each other. Moreover, it was shown that the patterns obtained within QED theory follow the motion of the wavepacket providing the correct periodicity of the motion, which cannot be captured by the semiclassical theory. In that case, the notion of the instantaneous electron density as the key quantity being probed in x-ray TRI for a sufficiently short pulse completely breaks down [40]. However, x-ray TRI from a single isolated hydrogen atom is not a realistic scenario. In practice, a sample contains several electrons and when a tunable pump pulse with broad energy bandwidth interacts with an $N$-electron system, one or few electrons participate in the formation of an electronic wavepacket and other electrons remain stationary. In such a situation, when an x-ray pulse scatters from a sample containing one or more non-stationary electrons and several stationary electrons, there is no way to know whether the scattering has taken place from the non-stationary electrons or from the stationary electrons and how the two scattering paths interfere with each other, i.e., interference between scattering from non-stationary and stationary electrons in the scattering process. Therefore, at this juncture it is important to analyze different types of contributions to the total scattering signal. The total signal can be decomposed into three main parts: first from stationary electrons, second from non-stationary electrons and third from the interference between non-stationary and stationary electrons. In the present work, 
we will analyze how these different contributions in an $N$-electron system contribute to the total scattering signal in both the theories (semiclassical and QED).

This paper is structured as follows. Section II discusses the formalism and results for x-ray TRI in the case of many-electron systems, where only one electron forms an electronic wavepacket and other electrons serve as stationary reference scatterers in both the theories. Effects of different parameters such as energy resolution of the detector, pulse duration and spectral bandwidth of the x-ray pulse etc. for the electron-electron interference in the scattering process are discussed in detail. Section III presents a numerical example of x-ray TRI for an electronic wavepacket in helium, where one electron forms a coherent superposition of one-electron eigenstates and the other electron remains stationary and serves as a reference scatterer. In this particular situation for helium, the role of the scattering interference is investigated. Conclusions and future outlook are presented in Sec. IV.

\section{THEORY}

Our investigations are based on the theory for x-ray TRI of electronic wavepacket motion as developed in Ref. [40]. Our equations are expressed in atomic units [41]. Under the assumptions that the probe pulse is centered at the energy of the incident pulse with very small energy width and the coherence length of the pulse is large in comparison to the size of the object, the expression for the DSP within the semiclassical theory is related to the Fourier transform of the instantaneous electron density, $\rho_{e}(\mathbf{x}, t)$, as follows [40]

$$
\frac{d P}{d \Omega}=\frac{d P_{e}}{d \Omega}\left|\int d^{3} x \rho_{e}(\mathbf{x}, t) e^{i \mathbf{Q} \cdot \mathbf{x}}\right|^{2} .
$$

Here, $\frac{d P_{e}}{d \Omega}$ is the DSP for a free electron and $\mathbf{Q}$ is the photon momentum transfer. Here, we have assumed that the x-ray pulse duration is shorter than the dynamical timescale of the electronic wavepacket. According to Eq. (1), the measured scattering pattern provides access to the instantaneous electron density as a function of the pump-probe delay time $t$.

Let us consider a scenario for time-resolved scattering of ultrashort x-rays in order to image the motion of a one-electron wavepacket in the presence of $N$ stationary electrons. In such a situation, the $N$ stationary electrons serve as reference scatterers in the total scattering signal. In this case, using the language of second quantization [42], the total 
electronic wavepacket can be written as

$$
|\Psi(t)\rangle=\hat{c}_{\varphi(t)}^{\dagger}\left|\Phi_{0}\right\rangle=\sum_{a} \alpha_{a} e^{-i \varepsilon_{a} t} \hat{c}_{a}^{\dagger}\left|\Phi_{0}\right\rangle
$$

with

$$
\hat{H}\left|\Phi_{0}\right\rangle=\left\{\sum_{i} \varepsilon_{i}\right\}\left|\Phi_{0}\right\rangle .
$$

Here, $\hat{c}_{p}^{\dagger}\left(\hat{c}_{p}\right)$ creates (annihilates) an electron in spin orbital $\left|\varphi_{p}\right\rangle$ and $\varepsilon_{p}$ is the orbital energy corresponding to $\left|\varphi_{p}\right\rangle$, i.e., $\hat{H}\left|\varphi_{p}\right\rangle=\varepsilon_{p}\left|\varphi_{p}\right\rangle$. $\hat{H}$ represents the electronic Hamiltonian at the mean-field level and $\left|\Phi_{0}\right\rangle$ is the unperturbed ground state of the $N$-electron system with the electrons filled to the Fermi level. Here and in the following, indices $p, q, r, s, \ldots$ are used for general spin orbitals (occupied or unoccupied). Occupied orbitals in $\left|\Phi_{0}\right\rangle$ are presented by indices $i, j, k, l, \ldots$, whereas unoccupied (virtual) orbitals are symbolized by $a, b, c, d, \ldots$.

We rewrite the key quantity in Eq. (1) in terms of the density operator

$$
\left|\int d^{3} x \rho_{e}(\mathbf{x}, t) e^{i \mathbf{Q} \cdot \mathbf{x}}\right|^{2}=\int d^{3} x \int d^{3} x^{\prime}\left\langle\Psi(t)\left|\hat{n}\left(\mathbf{x}^{\prime}\right)\right| \Psi(t)\right\rangle\langle\Psi(t)|\hat{n}(\mathbf{x})| \Psi(t)\rangle e^{i \mathbf{Q} \cdot\left(\mathbf{x}-\mathbf{x}^{\prime}\right)},
$$

with

$$
\hat{n}(\mathbf{x})=\hat{\psi}^{\dagger}(\mathbf{x}) \hat{\psi}(\mathbf{x})=\sum_{p q} \varphi_{p}^{\dagger}(\mathbf{x}) \varphi_{q}(\mathbf{x}) \hat{c}_{p}^{\dagger} \hat{c}_{q}
$$

Here, $\hat{n}(\mathbf{x})$ is the electron density operator, and the field operator $\hat{\psi}^{\dagger}(\mathbf{x})[\hat{\psi}(\mathbf{x})]$ creates (annihilates) an electron at position $\mathbf{x}$. Using the expression for the wavepacket as introduced in Eq. (2), Eq. (4) simplifies as follows

$$
\begin{aligned}
\left|\int d^{3} x \rho_{e}(\mathbf{x}, t) e^{i \mathbf{Q} \cdot \mathbf{x}}\right|^{2}= & \left|\sum_{i} \mathcal{L}_{i i}\right|^{2} \\
& +\sum_{i} \sum_{a b} \alpha_{a}^{*} \alpha_{b} e^{i\left(\varepsilon_{a}-\varepsilon_{b}\right) t}\left\{\mathcal{L}_{i i}^{*} \mathcal{L}_{a b}+\mathcal{L}_{i i} \mathcal{L}_{a b}^{*}\right\} \\
& +\left|\sum_{a b} \alpha_{a}^{*} \alpha_{b} \mathcal{L}_{a b} e^{i\left(\varepsilon_{a}-\varepsilon_{b}\right) t}\right|^{2},
\end{aligned}
$$

with

$$
\mathcal{L}_{p q}=\int d^{3} x \varphi_{p}^{\dagger}(\mathbf{x}) e^{i \mathbf{Q} \cdot \mathbf{x}} \varphi_{q}(\mathbf{x})
$$

and

$$
\mathcal{L}_{p q}^{*}=\int d^{3} x \varphi_{p}^{\dagger}(\mathbf{x}) e^{-i \mathbf{Q} \cdot \mathbf{x}} \varphi_{q}(\mathbf{x})
$$


Here, the right-hand side of Eq. (6a) provides the time-independent contributions due to scattering from the $N$ stationary electrons. The second term as shown in Eq. (6b) is due to the scattering interference between the $N$ stationary electrons and the non-stationary electron. The last time-dependent term in Eq. (6c) is solely due to scattering from the non-stationary electron.

On the other hand, in the full quantum theory of x-ray TRI, both matter and x-ray pulse are treated quantum mechanically and first-order time-dependent perturbation theory is employed for the interaction between matter and $\mathrm{x}$ rays. Here we assume that the probe pulse has a small bandwidth and a small angular spread so that the pixel assignment is well defined in the momentum space, the coherence length of the pulse is large in comparison to the size of the object, and the pulse duration should be sufficiently short to freeze the dynamics of the electronic wavepacket. Under these assumptions, the resulting expression for the DSP from a coherent, Gaussian x-ray pulse is [40]

$$
\begin{aligned}
\frac{d P}{d \Omega}= & \frac{d P_{e}}{d \Omega} \int_{0}^{\infty} d \omega_{\mathbf{k}_{s}} W_{\Delta E}\left(\omega_{\mathbf{k}_{s}}\right) \frac{\omega_{\mathbf{k}_{s}}}{\omega_{\mathbf{k}_{i n}}} \int_{-\infty}^{\infty} \frac{d \tau}{2 \pi} C(\tau) e^{-i\left(\omega_{\mathbf{k}_{s}}-\omega_{\mathbf{k}_{i n}}\right) \tau} \\
& \times \int d^{3} x \int d^{3} x^{\prime}\left\langle\Psi\left(t+\frac{\tau}{2}\right)\left|\hat{n}\left(\mathbf{x}^{\prime}\right) e^{-i \hat{H} \tau} \hat{n}(\mathbf{x})\right| \Psi\left(t-\frac{\tau}{2}\right)\right\rangle e^{i \mathbf{Q} \cdot\left(\mathbf{x}-\mathbf{x}^{\prime}\right)} .
\end{aligned}
$$

Here, $C(\tau)=\exp \left(-\frac{2 \ln 2 \tau^{2}}{\tau_{l}^{2}}\right)$ is a function of the pulse duration $\tau_{l}$. $\omega_{\mathbf{k}_{i n}}$ and $\omega_{\mathbf{k}_{s}}$ refer to the energy of the incident and scattered photon, respectively, while $W_{\Delta E}\left(\omega_{\mathbf{k}_{s}}\right)$ is a spectral window function centered at $\omega_{\mathbf{k}_{\text {in }}}$ with a width $\Delta E . W_{\Delta E}\left(\omega_{\mathbf{k}_{s}}\right)$ models the range of energies of the scattered photons accepted by the detector.

In the case of QED theory, an energy-resolved scattering process is considered for x-ray TRI. Therefore, any inelastic (Compton) scattering contributions due to excitations from the $N$ stationary electrons can be easily distinguished by utilizing the energy-resolving detector, if we assume that $\Delta E$ is small in comparison to the characteristic excitation energies of $\left|\Phi_{0}\right\rangle$. Hence, excitations from the $N$ stationary electrons are not considered in the following. Similarly, on using the expression for the wavepacket as introduced in Eq. (21), the key 
expression of Eq. (9) is simplified as follows

$$
\begin{aligned}
& \int d^{3} x \int d^{3} x^{\prime}\left\langle\Psi\left(t+\frac{\tau}{2}\right)\left|\hat{n}\left(\mathbf{x}^{\prime}\right) e^{-i \hat{H} \tau} \hat{n}(\mathbf{x})\right| \Psi\left(t-\frac{\tau}{2}\right)\right\rangle e^{i \mathbf{Q} \cdot\left(\mathbf{x}-\mathbf{x}^{\prime}\right)} \\
& =\left|\sum_{i} \mathcal{L}_{i i}\right|^{2} \\
& \quad+\sum_{i} \sum_{a b} \alpha_{a}^{*} \alpha_{b} e^{i\left(\varepsilon_{a}-\varepsilon_{b}\right) t}\left\{\mathcal{L}_{i i}^{*} \mathcal{L}_{a b} e^{-i\left(\varepsilon_{a}-\varepsilon_{b}\right) \frac{\tau}{2}}+\mathcal{L}_{i i} \mathcal{L}_{a b}^{*} e^{i\left(\varepsilon_{a}-\varepsilon_{b}\right) \frac{\tau}{2}}\right\} \\
& \quad+\sum_{a b} \sum_{c} \alpha_{a}^{*} \alpha_{b} \mathcal{L}_{a c}^{*} \mathcal{L}_{c b} e^{i\left(\varepsilon_{a}-\varepsilon_{b}\right) t} e^{i\left(\varepsilon_{a}+\varepsilon_{b}-2 \varepsilon_{c}\right) \frac{\tau}{2}} .
\end{aligned}
$$

Here, the first term, Eq. (10a), provides the time-independent contribution due to scattering from the $N$ stationary electrons, which is identical to Eq. (6a). The second term in Eq. (10b) is due to the scattering interference between the $N$ stationary electrons and the non-stationary electron, which seems different to the one shown in Eq. (6b). The last timedependent term, Eq. (10c), is again solely due to scattering from the non-stationary electron. The scattering contributions from the non-stationary electron, Eqs. (6c) and (10c), are not identical and provide completely different information about the electronic motion as shown in the case of a one-electron wavepacket in atomic hydrogen [40]. It is evident from Eqs. (66) and (10), that the first term in both the theories, Eqs. (6a) and (10a), provides identical scattering contributions to the total signal. In many systems of interest, the number of stationary electrons is large and therefore, the time-independent terms contribute a strong static background in the total signal. Due to the large number of the stationary electrons and one or few non-stationary electrons, the dominating time-dependent contributions in the total scattering signal are due to the scattering interference between stationary and non-stationary electrons (unless one is considering $\mathbf{Q}$ for which $\sum_{i} \mathcal{L}_{i i}$ is small). Therefore, it is crucial to analyze the scattering interference term, Eqs. (6b) and (10b), in both the theories.

On substituting Eqs. (6b) and (10b) in Eqs. (1) and (9), respectively, the expression for the scattering interference contribution to the DSP, $\frac{d P_{\text {int }}}{d \Omega}$, can be written as

$$
\frac{d P_{\mathrm{int}}}{d \Omega}=\frac{d P_{e}}{d \Omega} \sum_{i} \sum_{a b} \alpha_{a}^{*} \alpha_{b} e^{i\left(\varepsilon_{a}-\varepsilon_{b}\right) t}\left\{\mathcal{L}_{i i}^{*} \mathcal{L}_{a b}+\mathcal{L}_{i i} \mathcal{L}_{a b}^{*}\right\}
$$


in the semiclassical theory, and in the QED formalism can be expressed as

$$
\begin{aligned}
\frac{d P_{\text {int }}}{d \Omega}= & \frac{d P_{e}}{d \Omega} \int_{0}^{\infty} d \omega_{\mathbf{k}_{s}} W_{\Delta E}\left(\omega_{\mathbf{k}_{s}}\right) \frac{\omega_{\mathbf{k}_{s}}}{\omega_{\mathbf{k}_{i n}}} \frac{\tau_{l}}{\sqrt{8 \pi \ln 2}} \sum_{i} \sum_{a b} \alpha_{a}^{*} \alpha_{b} e^{i\left(\varepsilon_{a}-\varepsilon_{b}\right) t} \\
& \times\left\{\mathcal{L}_{i i}^{*} \mathcal{L}_{a b} e^{-\frac{\tau_{l}^{2}}{8 \ln 2}\left(\omega_{\mathbf{k}_{s}}-\omega_{\mathbf{k}_{i n}}+\left(\frac{\varepsilon_{a}-\varepsilon_{b}}{2}\right)\right)^{2}}+\mathcal{L}_{i i} \mathcal{L}_{a b}^{*} e^{-\frac{\tau_{l}^{2}}{8 \ln 2}\left(\omega_{\mathbf{k}_{s}}-\omega_{\mathbf{k}_{i n}}-\left(\frac{\varepsilon_{a}-\varepsilon_{b}}{2}\right)\right)^{2}}\right\} .
\end{aligned}
$$

It is important to analyze several aspects of both expressions, Eqs. (11) and (12), under different circumstances and properties of the probe pulse.

- The Gaussian distributions in Eq. (12) are centered at positions $\omega_{\mathbf{k}_{i n}}+\left(\frac{\varepsilon_{a}-\varepsilon_{b}}{2}\right)$ and $\omega_{\mathbf{k}_{i n}}-\left(\frac{\varepsilon_{a}-\varepsilon_{b}}{2}\right)$, and the width of the distributions is determined by $\tau_{l}$. Therefore, if $\Delta E$ is larger than the separation between the two distributions and the detector has a lower energy resolution than the energy spectral bandwidth of the x-ray pulse, then all the scattered photons are detected by the detector and $W_{\Delta E}$ is constant in the energy range contributing to the integral in Eq. (12). Hence, on performing the energy integral, Eq. (12) reduces to Eq. (11). However, when the energy resolution of the detector is poor, it is very difficult to assign a unique pixel in $Q$-space to the scattered photon in the detector and it may no longer be possible to filter out Compton scattering from the $N$ stationary electrons.

- If the probe pulse is very short in comparison to the dynamical timescale of the electronic motion, the $\tau$ dependent exponent in Eq. (10b) will reduce to unity. Therefore, if $\Delta E$ is smaller than the energy spectral width of the pulse and centered at the incident energy such that $\omega_{\mathbf{k}_{s}} \approx \omega_{\mathbf{k}_{i n}}$, the energy integral, Eq. (12) reduces to Eq. (11) and the scattering interference contributions from both the theories will be identical. However, an ultrashort pulse corresponds to a large energy spectral width due to the energy-time uncertainty relation. Therefore, the unavoidable energy bandwidth of the pulse causes an uncertainty in the momentum distribution of the incoming photon. In such a situation uniqueness of a pixel in $Q$-space is lost.

- In general, however, the probe pulse is not very short in comparison to the dynamical timescale of the electronic motion. In such situations the $\tau$ dependent exponent in Eq. (10b) will not reduce to unity and both expressions for the interference will provide different contributions to the total signal. 
In the following subsection, we present an example of one-electron wavepacket motion in helium. In this example, we apply both the approaches to compute time-resolved scattering patterns and analyze the contributions from the scattering interference to the total scattering signal.

\section{NUMERICAL RESULTS AND DISCUSSION}

A schematic scenario for probing an electronic wavepacket motion in helium is shown in Fig. 1. The ground state configuration for both the electrons is $1 \mathrm{~s}^{2}$. The ionization potential of the first electron is $24.59 \mathrm{eV}$ and $54.42 \mathrm{eV}$ for the second electron [43]. A pump pulse with broad energy bandwidth excites one of the electrons from the ground state configuration and prepares a coherent superposition of the 1s3d and 1s4f configurations with the projection of orbital angular momentum being equal to zero. The energy difference between the $1 \mathrm{~s}^{2}$ and $1 \mathrm{~s} 3 \mathrm{~d}$ configurations is $23.07 \mathrm{eV}$ and between the $1 \mathrm{~s} 3 \mathrm{~d}$ and $1 \mathrm{~s} 4 \mathrm{f}$ configurations is 0.66 eV (see Fig. 1). Therefore, the dynamical timescale of the electronic wavepacket motion, which is inversely related to the energy spacing between the eigenstates participating in the wavepacket, is $6.25 \mathrm{fs}$. The spatial extension of the wavepacket is $14-17 \AA$ along the $z$ axis and 7.5-9 $\AA$ along the $x$ and $y$ axes. It is known that for orbital angular momentum quantum number equal to or larger than two, the quantum defect is almost zero. Therefore, the electron in the $1 s$ orbital sees no shielding of the nuclear charge and the electron in the superposition of $3 d$ and $4 f$ orbitals sees complete shielding of the nuclear charge. Therefore, the wavefunction for the stationary electron in the $1 s$ orbital can be expressed in terms of the hydrogenic wavefunction with nuclear charge $\mathrm{Z}=2$. On the other hand, the wavefunction for the non-stationary electron in $3 d$ and $4 f$ orbitals as well as other higher-lying orbitals can be written in terms of the corresponding hydrogenic wavefunction with $\mathrm{Z}=1$. This type of procedure for treating the two-electron problem has already been used in the past and was successfully applied to describe different types of physical processes [44].

In order to compute time-dependent scattering patterns of the electronic wavepacket (cf. Fig. 1) as a function of the delay time in helium, we employ both the semiclassical and the QED approaches, i.e., Eqs. (11) and (9). Since the non-stationary and stationary electrons are energetically distinguishable (energy difference is around $23 \mathrm{eV}$ ) and an energy-resolved scattering process is considered with energy resolution at least equal to the unavoidable 


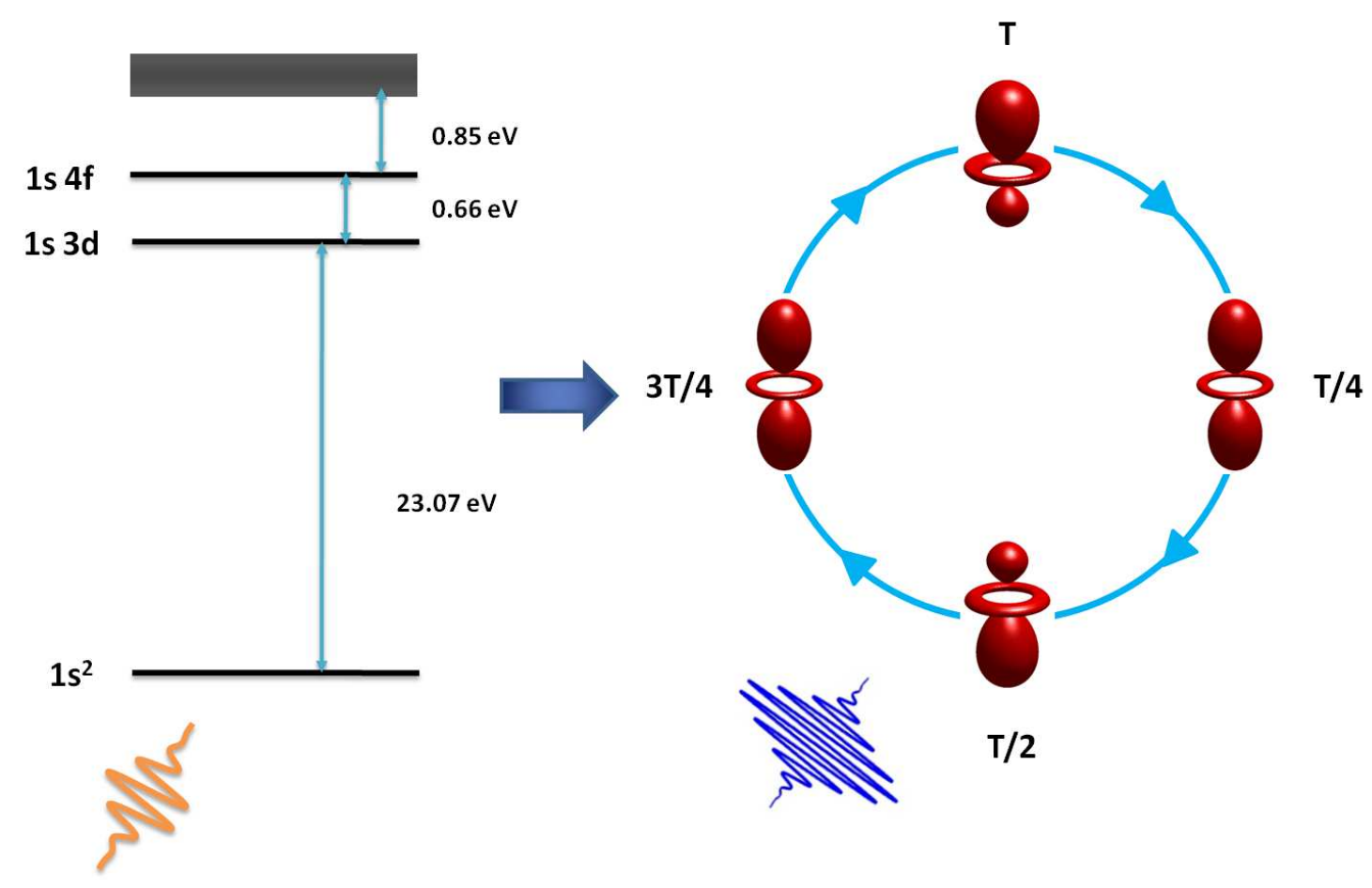

FIG. 1: A schematic scenario for probing an electronic wavepacket motion using ultrafast timeresolved x-ray scattering in helium atom. A pump pulse with broad energy bandwidth (indicated in orange) excites one of the electrons from the ground state and forms a coherent superposition of the 1s3d and 1s4f eigenstates with equal population. An isosurface of the electronic charge distribution of the wavepacket is shown (indicated in red), which undergoes periodic oscillation with oscillation period $\mathrm{T}=6.25$ fs. The dynamically evolving electronic charge distribution is probed by an ultrafast x-ray pulse (indicated in blue). By varying the pump-probe time delay, one obtains a series of scattering patterns that serve to image the electronic motion with atomic-scale spatio-temporal resolution.

spectral bandwidth of the probe pulse, any excitation from the stationary electron can be easily filtered out and therefore is not considered in the present case. The scattering operator $e^{i \mathbf{Q} \cdot \mathbf{x}}$ is expanded in terms of the spherical Bessel functions, $j_{l}(Q r)$, and spherical harmonics $Y_{m}^{l}(\theta, \phi)$ as

$$
e^{i \mathbf{Q} \cdot \mathbf{x}}=4 \pi \sum_{l, m} i^{l} j_{l}(Q r) Y_{m}^{l *}(\alpha, \beta) Y_{m}^{l}(\theta, \phi),
$$

where $r=|\mathbf{x}|$. After introducing hydrogenic wavefunctions, the expression in Eq. (91) fac- 
torizes into radial and angular parts. The angular part is given by

$$
\begin{aligned}
\int_{0}^{2 \pi} \int_{0}^{\pi} Y_{m_{1}}^{l_{1}}(\theta, \phi) Y_{m_{2}}^{l_{2}}(\theta, \phi) Y_{m_{3}}^{l_{3}}(\theta, \phi) \sin \theta d \theta d \phi & =\sqrt{\frac{\left(2 l_{1}+1\right)\left(2 l_{2}+1\right)\left(2 l_{3}+1\right)}{4 \pi}} \\
& \times\left(\begin{array}{lll}
l_{1} & l_{2} & l_{3} \\
0 & 0 & 0
\end{array}\right)\left(\begin{array}{ccc}
l_{1} & l_{2} & l_{3} \\
m_{1} & m_{2} & m_{3}
\end{array}\right)
\end{aligned}
$$

whereas the radial part is numerically integrated. To calculate the patterns as a function of the delay times, we used a Gaussian pulse of duration 1 fs with $4 \mathrm{keV}$ incoming photon energy, and assumed a Gaussian photon energy detection window of width $\Delta E=0.5 \mathrm{eV}$ for the detector. The patterns are calculated for $Q_{\max }=2 \AA^{-1}$ corresponding to a 3.14 $\AA$ spatial resolution and to a detection angle of scattered photons of up to $60^{\circ}$. In order to compute the scattering contribution from the non-stationary electron to the total scattering signal, all transitions induced during the scattering process within the energy detection window are computed [40]. Therefore, transition amplitudes from the eigenstates involved in the electronic wavepacket to all the electronic states within the detection range of $\Delta E$ are computed, which includes all types of multipole transitions allowed by the conservation of angular momentum and amounts to 22000 transition amplitudes. Also, the scattering patterns using semiclassical theory are calculated by convolution of the square of the Fourier transform of $\rho_{e}(\mathbf{x}, t)$ with an x-ray pulse of duration 1 fs.

Scattering patterns in the $Q_{x}-Q_{z}$ plane $\left(Q_{y}=0\right)$ as a function of the delay time at times $0, \mathrm{~T} / 4, \mathrm{~T} / 2,3 \mathrm{~T} / 4$, and $\mathrm{T}$ are depicted in Fig. 2. The time-dependent patterns shown in Fig. 2(a) are computed within QED theory using Eq. (9), whereas in Fig. 2(b) are computed within semiclassical theory using Eq. (11). It is evident from Fig. 2(a) that the patterns undergo spatial oscillation along $Q_{z}$ in momentum space and reflect the motion of the wavepacket along $z$ in real space (see Fig. 1). One of the striking features of the patterns shown in Fig. 2(a) is that when the charge distributions are symmetric, and corresponding patterns are asymmetric and vice versa, which can be understood as follows. The charge distributions are identical at delay times $\mathrm{T} / 4$ and $3 \mathrm{~T} / 4$, as may be seen in Fig. 1, while the electron clouds move in opposite directions at the two times. At time $\mathrm{T} / 4$, the flow of the electron cloud is downwards, whereas at time $3 \mathrm{~T} / 4$ the flow is upwards. This is reflected by their corresponding patterns. Therefore, the patterns calculated within the QED theory capture the dynamics of the momentum distribution of the wavepacket. As a consequence, the apparent motions of the charge distributions and of the scattering patterns are shifted 
(a)
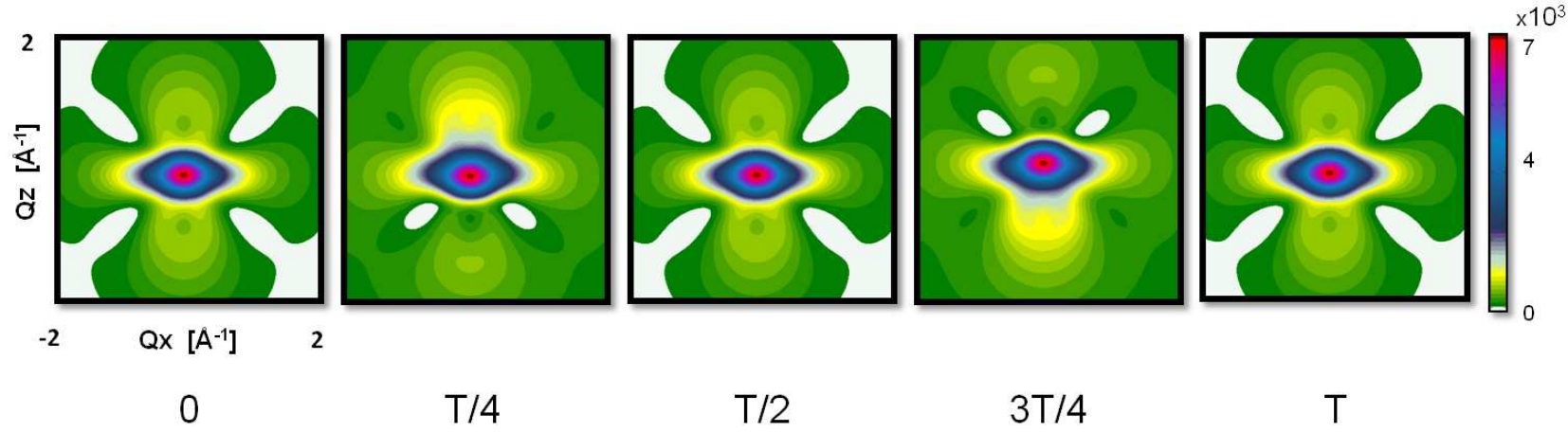

(b)

T/4

T/2

3T/4 T
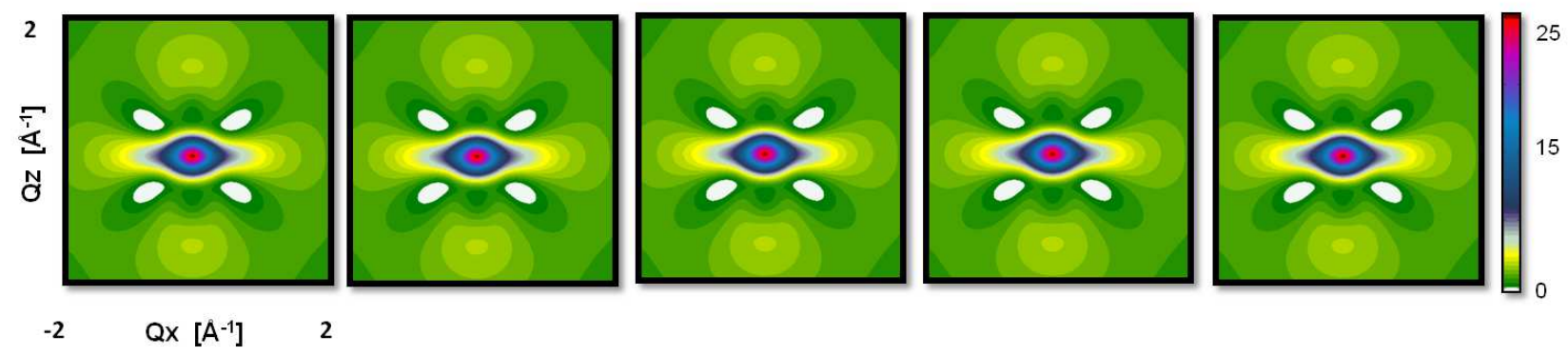

FIG. 2: Scattering patterns in the $Q_{x}-Q_{z}$ plane $\left(Q_{y}=0\right)$ of helium (cf. Fig. 1). Scattering patterns obtained using (a) QED theory, i.e., Eq. (9) and (b) semiclassical theory, i.e., Eq. (11) at pump-probe delay times $0, \mathrm{~T} / 4, \mathrm{~T} / 2,3 \mathrm{~T} / 4$, and $\mathrm{T}$, where the oscillation period of the electronic wavepacket is $\mathrm{T}=6.25 \mathrm{fs}$.

by $90^{\circ}$. On the other hand, the patterns shown in Fig. 2(b) do not change significantly as a function of the delay time.

In order to understand the scattering from the non-stationary electron, the scattering patterns corresponding to the non-stationary electron are shown in Fig. 3 in the $Q_{x}-Q_{z}$ plane $\left(Q_{y}=0\right)$ as a function of the delay time. The patterns shown in Fig. 3(a) are obtained using Eq. (10c) and in Fig. 3(b) are obtained using Eq. (6c). It is evident from Fig. 3(a) that the scattering patterns undergo oscillations as a function of the delay time. The scattering patterns shown in Fig. 3(b) are localized in the low $Q$ region, which reflects the spatial extension of the electronic charge distribution of the wavepacket and also undergo changes as a function of the delay time, but do not display oscillations as the charge distribution oscillates. Hence, patterns obtained using semiclassical theory provide half of the actual period of the motion as the patterns start repeating themselves in half of the actual time of the motion. 
We now investigate the contribution from the scattering interference between the stationary and non-stationary electrons to the total scattering signal in both the theories. On re-writing Eqs. (7) and (8) in the case of helium, we find that

$$
\mathcal{L}_{1 s 1 s}=\int d^{3} x \varphi_{1 s}^{\dagger}(\mathbf{x}) e^{i \mathbf{Q} \cdot \mathbf{x}} \varphi_{1 s}(\mathbf{x})
$$

is a purely real number, as is evident from Eqs. (13) and (14), whereas

$$
\mathcal{L}_{3 d 4 f}=\int d^{3} x \varphi_{3 d}^{\dagger}(\mathbf{x}) e^{i \mathbf{Q} \cdot \mathbf{x}} \varphi_{4 f}(\mathbf{x})
$$

is a purely imaginary number, which also follows from Eqs. (13) and (14). Therefore,

$$
\mathcal{L}_{1 s 1 s}=\mathcal{L}_{1 s 1 s}^{*}
$$

and

$$
\mathcal{L}_{3 d 4 f}=-\mathcal{L}_{3 d 4 f}^{*}
$$

In a similar way we can write $\mathcal{L}_{3 d 3 d}=\mathcal{L}_{3 d 3 d}^{*}$, and $\mathcal{L}_{4 f 4 f}=\mathcal{L}_{4 f 4 f}^{*}$, which are both real. On substituting the contributions of the $\mathcal{L}$ 's in Eq. (6b), the contribution from the scattering interference within the semiclassical theory can be written as

$$
\sum_{a=3 d, 4 f} \sum_{b=3 d, 4 f} \alpha_{a}^{*} \alpha_{b} e^{i\left(\varepsilon_{a}-\varepsilon_{b}\right) t}\left\{\mathcal{L}_{1 s 1 s}^{*} \mathcal{L}_{a b}+\mathcal{L}_{1 s 1 s} \mathcal{L}_{a b}^{*}\right\}
$$

which can be further decomposed into two parts: a time-dependent scattering interference contribution

$$
\begin{aligned}
& \alpha_{3 d}^{*} \alpha_{4 f} e^{i\left(\varepsilon_{3 d}-\varepsilon_{4 f}\right) t}\left\{\mathcal{L}_{1 s 1 s}^{*} \mathcal{L}_{3 d 4 f}+\mathcal{L}_{1 s 1 s} \mathcal{L}_{3 d 4 f}^{*}\right\}+\alpha_{4 f}^{*} \alpha_{3 d} e^{i\left(\varepsilon_{4 f}-\varepsilon_{3 d}\right) t}\left\{\mathcal{L}_{1 s 1 s}^{*} \mathcal{L}_{4 f 3 d}+\mathcal{L}_{1 s 1 s} \mathcal{L}_{4 f 3 d}^{*}\right\} \\
& =\alpha_{3 d}^{*} \alpha_{4 f} e^{i\left(\varepsilon_{3 d}-\varepsilon_{4 f}\right) t} \mathcal{L}_{1 s 1 s}\left\{\mathcal{L}_{3 d 4 f}-\mathcal{L}_{3 d 4 f}\right\}+\alpha_{4 f}^{*} \alpha_{3 d} e^{i\left(\varepsilon_{4 f}-\varepsilon_{3 d}\right) t} \mathcal{L}_{1 s 1 s}\left\{\mathcal{L}_{4 f 3 d}-\mathcal{L}_{4 f 3 d}\right\}=0
\end{aligned}
$$

which is zero, and a time-independent scattering interference contribution

$$
2 \mathcal{L}_{1 s 1 s}\left[\left|\alpha_{3 d}\right|^{2} \mathcal{L}_{3 d 3 d}+\left|\alpha_{4 f}\right|^{2} \mathcal{L}_{4 f 4 f}\right]
$$

Similarly on substituting the contributions of the $\mathcal{L}$ 's in Eq. (10b), the contribution from the scattering interference within the QED theory can be written as the sum of two parts: a time-dependent scattering interference contribution

$$
\begin{aligned}
& \alpha_{3 d}^{*} \alpha_{4 f} e^{i\left(\varepsilon_{3 d}-\varepsilon_{4 f}\right) t} \mathcal{L}_{1 s 1 s} \mathcal{L}_{3 d 4 f}\left\{e^{-i\left(\varepsilon_{3 d}-\varepsilon_{4 f}\right) \frac{\tau}{2}}-e^{i\left(\varepsilon_{3 d}-\varepsilon_{4 f}\right) \frac{\tau}{2}}\right\} \\
& \quad+\alpha_{4 f}^{*} \alpha_{3 d} e^{i\left(\varepsilon_{4 f}-\varepsilon_{3 d}\right) t} \mathcal{L}_{1 s 1 s} \mathcal{L}_{4 f 3 d}\left\{e^{-i\left(\varepsilon_{4 f}-\varepsilon_{3 d}\right) \frac{\tau}{2}}-e^{i\left(\varepsilon_{4 f}-\varepsilon_{3 d}\right) \frac{\tau}{2}}\right\},
\end{aligned}
$$


which is non-zero, and a time-independent scattering interference contribution

$$
2 \mathcal{L}_{1 s 1 s}\left[\left|\alpha_{3 d}\right|^{2} \mathcal{L}_{3 d 3 d}+\left|\alpha_{4 f}\right|^{2} \mathcal{L}_{4 f 4 f}\right]
$$

which is identical to the one obtained within semiclassical theory. We rewrite the timedependent interference contribution, Eq (22), to the leading nonvanishing order in $\tau$ as

$$
i \mathcal{L}_{1 s 1 s}\left(\varepsilon_{4 f}-\varepsilon_{3 d}\right) \tau\left[\alpha_{3 d}^{*} \alpha_{4 f} \mathcal{L}_{3 d 4 f} e^{i\left(\varepsilon_{3 d}-\varepsilon_{4 f}\right) t}-\alpha_{4 f}^{*} \alpha_{3 d} \mathcal{L}_{4 f 3 d} e^{i\left(\varepsilon_{4 f}-\varepsilon_{3 d}\right) t}\right] \neq 0 .
$$

Therefore, for the particular combination of orbitals involved in the one-electron wavepacket and the orbital corresponding to the stationary electron in helium (see Fig. 1), the timedependent scattering interference between non-stationary and stationary orbitals is zero within the semiclassical theory and negligibly small in comparison to the total scattering signal in the QED theory. However, the time-independent scattering interference between the orbitals, i.e., the interference between $1 s$ and $3 d$ orbitals and $1 s$ and $4 f$ orbitals, contributes equally to the total scattering signal in both the theories, as is evident from Eqs. (21) and (23). The reason why the time-dependent scattering interference contribution to the total signal is so small in QED theory can be understood as follows: For probing the ultrafast motion, one has to satisfy $\Delta \varepsilon \tau \ll 1$, where $\Delta \varepsilon=\varepsilon_{4 f}-\varepsilon_{3 d}$ is the characteristic energy scale of the electronic wavepacket, i.e., the pulse duration of the probe pulse should be much smaller than the characteristic timescale of the motion. Thus, the contribution from Eq. (24), which is proportional to $\Delta \varepsilon \tau$, is suppressed.

On comparing the scattering contribution from the non-stationary electron with respect to total scattering signal, i.e., comparing Figs. 3)(a) and 2(a), one can easily distinguish the scattering contributions from the stationary electron and the interferences to the total scattering patterns in the QED theory. In Fig. 2(a), the broadening of the scattering signal in the high $Q$ region is the reflection of the contribution from the time-independent scattering interference between orbitals, whereas the wing type structures along the diagonal, which change as a function of the delay time, are a reflection of the contribution from the timedependent scattering interference between orbitals, which is weak in comparison to the total scattering signal. On the other hand, on comparing the patterns shown in Figs. 2(b) and 3(b), one can only observe the broadening of scattering patterns, which reflects the contribution from the time-independent scattering interference between orbitals.

Therefore, the patterns within the semiclassical theory are dominated by the scattering contribution from the time-independent interference between orbitals, and are not changing 
(a)

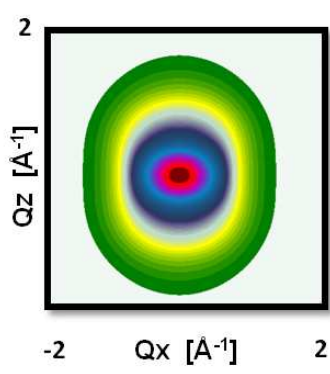

(b)

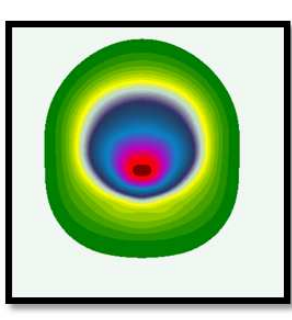

$\mathrm{T} / 4$

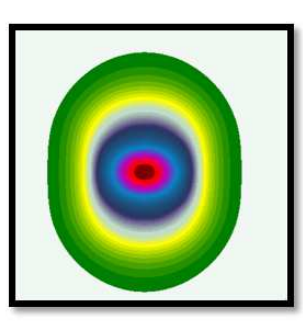

$\mathrm{T} / 2$

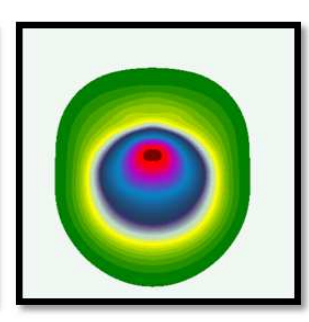

$3 T / 4$

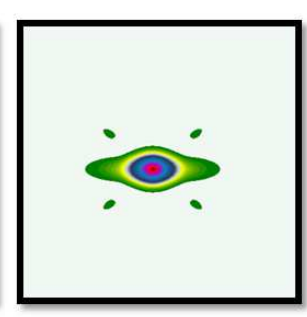

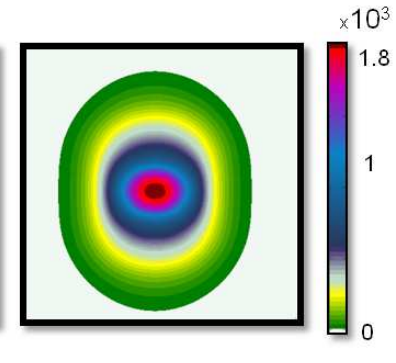

$\mathrm{T}$
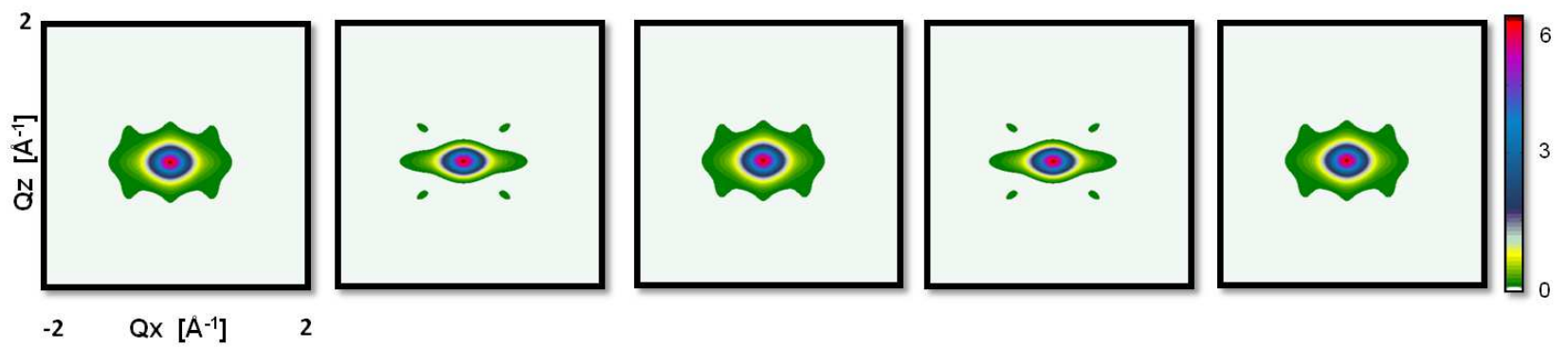

FIG. 3: Scattering contributions from the non-stationary electron to the total scattering patterns in the $Q_{x}-Q_{z}$ plane $\left(Q_{y}=0\right)$ in helium. Scattering patterns obtained using (a) Eq. (10c) and (b) Eq. (6c) at pump-probe delay times $0, \mathrm{~T} / 4, \mathrm{~T} / 2,3 \mathrm{~T} / 4$, and $\mathrm{T}$, where the oscillation period of the electronic wavepacket is $\mathrm{T}=6.25 \mathrm{fs}$.

significantly as a function of the delay time. In contrast, the patterns within the QED theory are dominated by the scattering contributions from the non-stationary electron due to Compton scattering within the finite energy detection range of the detector.

\section{CONCLUSIONS}

This work is devoted to understanding ultrafast time-resolved x-ray scattering from a sample containing a mixture of a non-stationary electron in the form of a one-electron wavepacket and one or more stationary electrons using the semiclassical theory and the QED theory of light-matter interaction. The contributions of the scattering interference between the non-stationary and the stationary electrons to the total time-dependent scattering signal are investigated in both the theories. Our investigations are based on the recent theory for time-resolved x-ray scattering to image the electronic wavepacket motion [40]. First, 
we investigated different scattering contributions to the total scattering signal in both the theories and showed that the total signal can be decomposed into three main scattering contributions: first from the stationary electrons, second from the non-stationary electron and third from the interference between stationary and non-stationary electrons. In both the theories, the scattering contributions from the stationary electrons to the signal are identical, whereas scattering contributions from the non-stationary electron are completely different. In the QED theory, the scattering contributions from the interference depend on the energy resolution of the detector and the x-ray pulse duration. Therefore, in case of negligible energy resolution or extremely short pulses, QED theory provides identical contributions for the scattering interference as one obtaines in the semiclassical theory. On the other hand, if the pulse duration is not very short in comparison to the dynamical timescale of the motion and if the energy resolution is sufficiently high, the scattering interference in the QED theory does not provide identical result to the one obtaines in the semiclassical theory. It is important to note that most organic molecules and proteins contain mainly hydrogen, carbon, nitrogen and oxygen atoms and in such cases the scattering signal is dominated by the scattering from carbon, nitrogen and oxygen atoms. In these atoms, only two electrons are deeply bound core electrons, whereas other electrons are loosely bound valence electrons. When a pump pulse with broad bandwidth initiates excitation in such atoms it might be possible that more than one electron participates in the formation of the electronic wavepacket. In such situations, the time-dependent scattering signal would be dominated by the scattering contributions from the non-stationary electrons rather than the stationary and the interference contributions.

Both the theories for light-matter interaction are illustrated by means of calculating the time-dependent scattering patterns for a one-electron wavepacket in helium. In helium, the pump pulse excites one of the electrons from the ground state and prepares an electronic wavepacket as a coherent superposition of the 1s3d and 1s4f eigenstates. The scattering patterns are computed for the non-stationary electron in the presence of a stationary electron. The time-dependent interference between the stationary and non-stationary electrons within the semiclassical theory is zero, and it is quite small in comparison to the total scattering signal in the QED theory. However, the time-independent interference between the stationary and non-stationary electrons contributes identically to the total signal in both the theories. The patterns are dominated by the scattering contribution from the time-independent inter- 
ference within the semiclassical theory, whereas the patterns are dominated by the scattering contributions from the non-stationary electron due to Compton scattering within the QED theory. Henceforth, the dynamical features of the patterns cannot be captured within semiclassical theory. We expect that our present analysis of TRI using ultrafast x-ray scattering will find several important applications for exploring ultrafast dynamics in nature. With the recent advent of novel light sources, we also believe that our findings will shed light on ultrafast electronic motion, for example, in atoms, molecules and biological systems [45 49].

\section{Acknowledgments}

We thank Jan Malte Slowik for careful reading of the manuscript.

[1] F. Krausz and M. Ivanov, Rev. Mod. Phys. 81, 163 (2009).

[2] P. H. Bucksbaum, Science 317, 766 (2007).

[3] P. B. Corkum and F. Krausz, Nature Physics 3, 381 (2007).

[4] O. Smirnova, Y. Mairesse, S. Patchkovskii, N. Dudovich, D. Villeneuve, P. Corkum, and M. Y. Ivanov, Nature 460, 972 (2009).

[5] E. Goulielmakis, Z. H. Loh, A. Wirth, R. Santra, N. Rohringer, V. S. Yakovlev, S. Zherebtsov, T. Pfeifer, A. M. Azzeer, M. F. Kling, S. R. Leone, and F. Krausz, Nature 466, 739 (2010).

[6] J. Breidbach and L. S. Cederbaum, J. Chem. Phys. 118, 3983 (2003).

[7] A. I. Kuleff, J. Breidbach, and L. S. Cederbaum, J. Chem. Phys. 123, 044111 (2005).

[8] F. Remacle and R. D. Levine, Proc. Natl. Acad. Sci. U.S.A 103, 6793 (2006).

[9] A. D. Dutoi and L. S. Cederbaum, J. Phys. Chem. Lett. 2, 2300 (2011).

[10] J. P. Bergsma, M. H. Coladonato, P. M. Edelsten, J. D. Kahn, K. R. Wilson, and D. R. Fredkin, Journal of Chemical Physics 84, 6151 (1986).

[11] S. Bratos, F. Mirloup, R. Vuilleumier, M. Wulff, and A. Plech, Chemical Physics 304, 245 (2004).

[12] A. Debnarova, S. Techert, and S. Schmatz, J. Chem. Phys. 125, 224101 (2006).

[13] A. Debnarova, S. Techert, and S. Schmatz, J. Chem. Phys. 133, 124309 (2010).

[14] P. Emma et al., Nature Photonics 4, 641 (2010). 
[15] T. Ishikawa et al., Nature Photonics 6, 540 (2012).

[16] A. Rousse, C. Rischel, and J. C. Gauthier, Rev. Mod. Phys. 73, 17 (2001).

[17] I. McKinnie and H. Kapteyn, Nature Photonics 4, 149 (2010).

[18] T. Popmintchev, M. C. Chen, D. Popmintchev, P. Arpin, S. Brown, S. Ališauskas, G. Andriukaitis, T. Balčiunas, O. D. Mücke, A. Pugzlys, A. Baltuska, B. Shim, S. E. Schrauth, A. Gaeta, C. Hernandez-Garcia, L. Plaja, A. Becker, A. Jaron-Becker, M. M. Murnane, and H. C. Kapteyn, Science 336, 1287 (2012).

[19] M. J. J. Vrakking and T. Elsaesser, Nature Photonics 6, 645 (2012).

[20] D. Pile, Nature Photonics 5, 456 (2011).

[21] P. Emma, K. Bane, M. Cornacchia, Z. Huang, H. Schlarb, G. Stupakov, and D. Walz, Phys. Rev. Lett. 92, 74801 (2004).

[22] A. A. Zholents and W. M. Fawley, Phys. Rev. Lett. 92, 224801 (2004).

[23] L. Young, E. P. Kanter, B. Krässig, Y. Li, A. M. March, S. T. Pratt, R. Santra, S. H. Southworth, N. Rohringer, L. F. DiMauro, G. Doumy, C. A. Roedig, N. Berrah, L. Fang, M. Hoener, P. H. Bucksbaum, J. P. Cryan, S. Ghimire, J. M. Glownia, D. A. Reis, J. D. Bozek, C. Bostedt, and M. Messerschmidt, Nature 466, 56 (2010).

[24] N. Rohringer, D. Ryan, R. A. London, M. Purvis, F. Albert, J. Dunn, J. Bozek, C. Bostedt, A. Graf, R. Hill, , S. P. Hau-Riege, and J. J. Rocca, Nature 481, 488 (2012).

[25] M. Hoener et al., Phys. Rev. Lett. 104, 253002 (2010).

[26] N. Berrah, L. Fang, B. Murphy, T. Osipov, K. Ueda, E. Kukk, R. Feifel, P. van der Meulen, P. Salen, H. T. Schmidt, R. D. Thomas, M. Larsson, R. Richter, K. C. Prince, J. D. Bozek, C. Bostedt, S. Wada, M. N. Piancastelli, M. Tashiro, and M. Ehara, Proc. Natl. Acad. Sci. U.S.A 108, 16912 (2011).

[27] H. Thomas, A. Helal, K. Hoffmann, N. Kandadai, J. Keto, J. Andreasson, B. Iwan, M. Seibert, N. Timneanu, J. Hajdu, M. Adolph, T. Gorkhover, D. Rupp, S. Schorb, T. Moller, G. Doumy, L. F. DiMauro, M. Hoener, B. Murphy, N. Berrah, M. Messerschmidt, J. Bozek, C. Bostedt, and T. Ditmire, Phys. Rev. Lett. 108, 133401 (2012).

[28] H. N. Chapman et al., Nature 470, 73 (2011).

[29] M. M. Seibert et al., Nature 470, 78 (2011).

[30] S. M. Vinko et al., Nature 482, 59 (2012).

[31] O. Ciricosta et al., Phys. Rev. Lett. 109, 65002 (2012). 
[32] W. C. Rontgen, Science 3, 227 (1896).

[33] J. Als-Nielsen and D. McMorrow, Elements of modern X-ray physics, Wiley, New York, 2011.

[34] S. Bratos, F. Mirloup, R. Vuilleumier, and M. Wulff, J. Chem. Phys. 116, 10615 (2002).

[35] A. M. Lindenberg, Y. Acremann, D. P. Lowney, P. A. Heimann, T. K. Allison, T. Matthews, and R. W. Falcone, J. Chem. Phys. 122, 204507 (2005).

[36] J. Hallmann, W. Morgenroth, C. Paulmann, J. Davaasambuu, Q. Kong, M. Wulff, and S. Techert, Journal of the American Chemical Society 131, 15018 (2009).

[37] H. Ihee, M. Wulff, J. Kim, and S. Adachi, International Reviews in Physical Chemistry 29, $453(2010)$.

[38] M. Ben-Nun, T. J. Martínez, P. M. Weber, and K. R. Wilson, Chem. Phys. Lett. 262, 405 (1996).

[39] A. Kirrander, J. Chem. Phys. 137, 154310 (2012).

[40] G. Dixit, O. Vendrell, and R. Santra, Proc. Natl. Acad. Sci. U.S.A 109, 11636 (2012).

[41] A. Szabo and N. S. Ostlund, Modern quantum chemistry: introduction to advanced electronic structure theory, Dover Publications, 1996.

[42] A. L. Fetter and J. D. Walecka, Quantum theory of many-particle systems, Dover Pubns, 2003.

[43] Y. Ralchenko, F. C. Jou, D. E. Kelleher, A. E. Kramida, A. Musgrove, J. Reader, W. L. Wiese, and K. Olsen, NIST Atomic Spectra Database, NIST Standard Reference Database (2006).

[44] H. A. Bethe and E. E. Salpeter, Quantum mechanics of one-and two-electron atoms, Plenum Publishing Corporation, 1977.

[45] S. Haessler, J. Caillat, W. Boutu, C. Giovanetti-Teixeira, T. Ruchon, T. Auguste, Z. Diveki, P. Breger, A. Maquet, B. Carré, R. Taeb, and P. Salires, Nature Physics 6, 200 (2010).

[46] P. Tzallas, E. Skantzakis, L. A. A. Nikolopoulos, G. D. Tsakiris, and D. Charalambidis, Nature Physics 7, 781 (2011).

[47] P. Hockett, C. Z. Bisgaard, O. J. Clarkin, and A. Stolow, Nature Physics 7, 612 (2011).

[48] H. Niikura, F. Legare, R. Hasbani, M. Y. Ivanov, D. M. Villeneuve, and P. B. Corkum, Nature 421, 826 (2002).

[49] H. Ihee, M. Lorenc, T. K. Kim, Q. Y. Kong, M. Cammarata, J. H. Lee, S. Bratos, and M. Wulff, Science 309, 1223 (2005). 